Provided for non-commercial research and education use. Not for reproduction, distribution or commercial use.

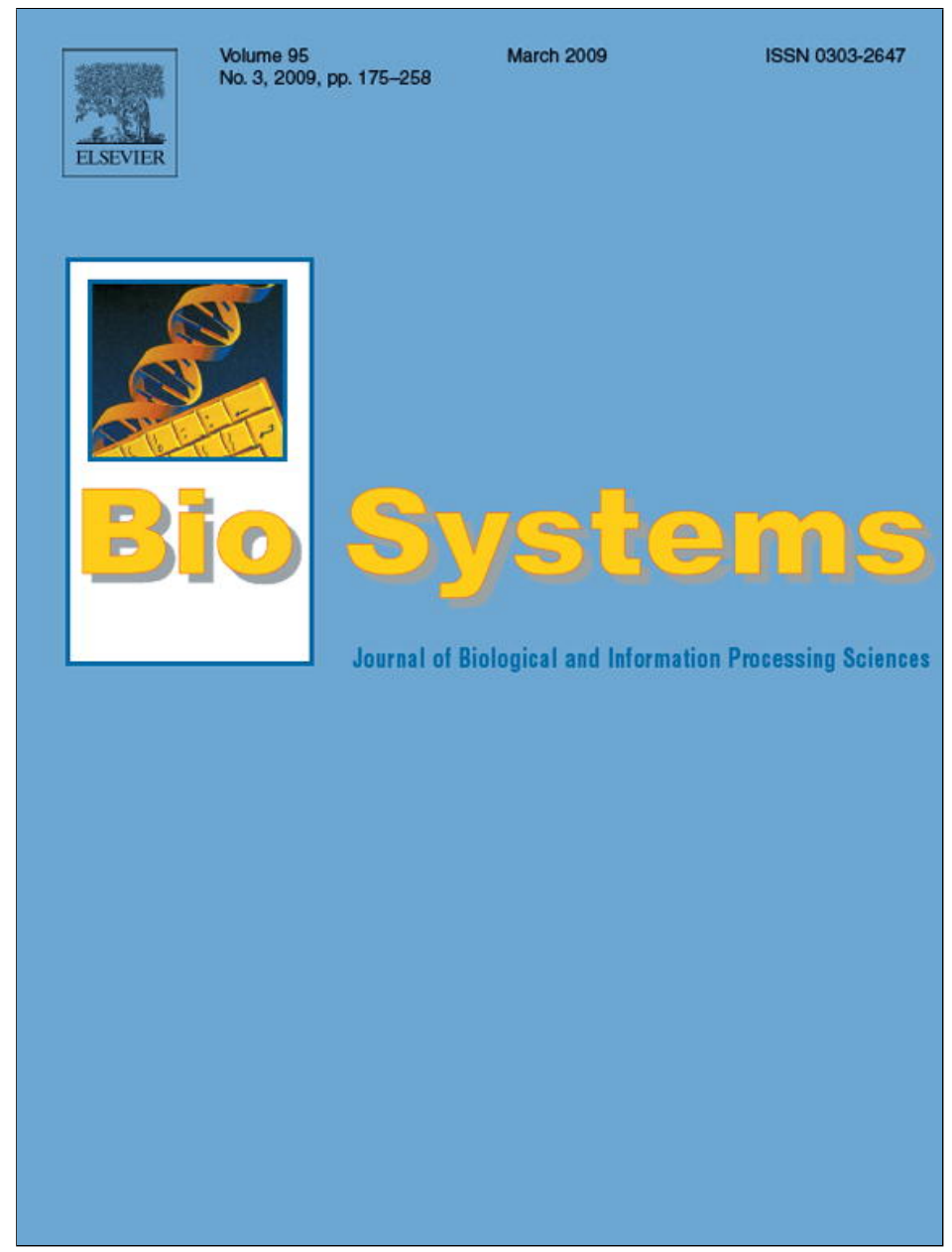

This article appeared in a journal published by Elsevier. The attached copy is furnished to the author for internal non-commercial research and education use, including for instruction at the authors institution and sharing with colleagues.

Other uses, including reproduction and distribution, or selling or licensing copies, or posting to personal, institutional or third party websites are prohibited.

In most cases authors are permitted to post their version of the article (e.g. in Word or Tex form) to their personal website or institutional repository. Authors requiring further information regarding Elsevier's archiving and manuscript policies are encouraged to visit:

http://www.elsevier.com/copyright 


\title{
Natural hierarchy emerges from energy dispersal
}

\author{
Arto Annila a,b,c,*, Esa Kuismanen ${ }^{c}$ \\ a Department of Physics, University of Helsinki, FI-00014 Helsinki, Finland \\ ${ }^{\mathrm{b}}$ Institute of Biotechnology, University of Helsinki, FI-00014 Helsinki, Finland

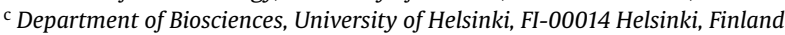

\section{A R T I C L E I N F O}

\section{Article history:}

Received 15 August 2008

Received in revised form 27 October 2008

Accepted 28 October 2008

\section{Keywords:}

Energy transduction

Entropy

Evolution

Free energy

Hierarchy

Life

Natural process

Natural selection

Self-organization

Statistical mechanics

Thermodynamics

\begin{abstract}
A B S T R A C T
Hierarchical organization of 'systems within systems' is an apparent characteristic of nature. For many biotic and abiotic systems it is known how the nested structural and functional order builds up, yet the general principle why matter evolves to hierarchies has remained unfamiliar to many. We clarify that increasingly larger integrated systems result from the quest to decrease free energy according to the 2nd law of thermodynamics. The argumentation is based on the recently derived equation of motion for natural processes. Hierarchically organized energy transduction machinery emerges naturally when it provides increased rates of energy dispersal. Likewise, a hierarchical system will dismantle into its constituents when they as independent systems will provide higher rates of entropy increase. Since energy flows via interactions, decreasing strengths of interactions over increasingly larges lengths scales mark natural boundaries for nested integrated systems.
\end{abstract}

(c) 2008 Elsevier Ireland Ltd. All rights reserved.

\section{Introduction}

Nature is hierarchical in its organization. For example, cells arrange into organs that make an organism, organisms in turn add up to an ecosystem and ecosystems total the global biosphere. The nested pattern is also found when descending downward in scale. For example, a eukaryotic cell houses cellular organelles that contain molecular complexes assembled from molecules that are, in turn, composed of atoms and so on. The structural and functional self-similarity of nature has become more and more evident to us as subatomic details and cosmic depths have reached our sight. Structures set the length scale whereas functions, in general motions, mark the time scale. In many cases it is understood how matter transforms from basic constituents to larger assemblies and vice versa. However the basic principle, why matter organizes as 'systems within systems' and why in other cases systems disassemble to constituents, has remained obscure.

The simple question concerning the driving force looks for a reason. Contemporary consensus recognizes on one hand natural

Corresponding author at: Department of Physics, University of Helsinki, POB 64, FI-00014 Helsinki, Finland. Tel.: +358 9191 50629; fax: +358 919150639. E-mail address: arto.annila@helsinki.fi (A. Annila). selection within the theory of evolution (Darwin, 1859) as the ubiquitous imperative that guides biotic processes and on the other hand the 2nd law of thermodynamics (Boltzmann, 1886; Carnot, 1824; Clausius, 1879; Gibbs, 1876) as another universal law that directs abiotic processes. Today these two driving forces are often perceived as opposing, one as constructive and the other as destructive. However, recently it was shown, by a first-principle derivation that they are in fact one and the same law (Sharma and Annila, 2007). The fundamental law simply states that energy differences diminish via flows of energy. To abolish energy gradients most rapidly the flows funnel through those mechanisms that level differences most effectively.

The universal law finds no demarcation line between animate and inanimate. Both systems evolve by flows of energy toward stationary states with respect to their surroundings. Those systems that couple effectively to a high-energy source, e.g. solar radiation, are mainly referred to as biological, whereas those that settle in low-energy ambient surroundings, e.g. cold space, are mostly referred to as abiotic. Thus, the law of energy dispersal itself is universal but the nature of a particular system depends on its surrounding energy density. Many animate mechanisms of energy transduction, such as plant and animal species, are complex whereas many inanimate means of energy transport, such as conductors and conveyers, are simple but the principle is the 
same. Since the transduction mechanisms are themselves repositories of energy, they are themselves subject to degradation by other means to yield lower quality gradients. This holistic interdependence among all entities in thermodynamics system is described by the 2nd law.

In this study the 2nd law of thermodynamics is used to explain why evolution emerges with functional, self-organizational order, not the details how a specific system evolves to hierarchy. The argumentation is based on the recently derived equation of motion (Sharma and Annila, 2007) for natural processes (Kondepudi and Prigogine, 1998) that has also been linked with (Kaila and Annila, 2008) the principle of least action (Maupertuis, 1746) and with Gyarmati's principle (Gyarmati, 1970; Lucia, 1995). The formalism has been applied to account for diverse phenomena and regularities in nature (Annila and Annila, in press; Grönholm and Annila, 2007; Jaakkola et al., 2008a,b; Karnani and Annila, 2008; Würtz and Annila, in press). Here, hierarchical organizations are regarded by the same principle as powerful machineries for energy dispersal. According to this view the hierarchically integrated systems have emerged and evolved increasingly more effective in leveling differences and gradients in energy densities. The presented account is in agreement with the earlier reasoning, namely that various evolutionary processes naturally emerge with hierarchical organization (Brooks and Wiley, 1986; Brooks, 2000; Chaisson, 2001; Lorenz, 2002; Matsuno and Salthe, 2002; Matsuno and Swenson, 1999; Salthe, 1985, 1993, 2004; Schneider and Kay, 1994; Ulanowicz and Hannon, 1987; Weber et al., 1989). Thus, the following description that emphasizes energy dispersal by gradient reduction as the motive force behind the hierarchical organization is not new but equivalent to the maximum entropy production principle (Dewar, 2003; Jaynes, 1957; Lucia, 2008). The presented description is based on the thermodynamic entropy (Kaila and Annila, 2008; Sharma and Annila, 2007) rather than on the informational entropy (Shannon, 1948).

\section{Forces and flows}

A difference in energy density is a thermodynamic driving force. Since energy is bound in various forms, also forces manifest in various ways. However, according to Gibbs (1876), any particular energy density of a population $N_{j}$ of entities $j$ can be denoted concisely by $\Phi_{j}=N_{j} \exp \left(G_{j} / R T\right)$ where the free energy $G_{j}$ is given relative to the average energy $R T$, e.g. per mole. The corresponding potential (Atkins, 1998) is $\mu_{j} / R T=\ln \left[N_{j} \exp \left(G_{j} / R T\right)\right]$. All potential energy differences and gradients relative to $R T$, that an entity $j$ is subject to, can be denoted by $\nabla\left(\mu_{j} / R T\right)$. The concise notation contains a spatial difference in numbers $N_{j}$, i.e., a concentration gradient that will lead to diffusion. Likewise, a temperature difference will lead to heat flows. Chemical potential energy difference $\nabla \mu_{j}=\sum \mu_{k}-\mu_{j}$ is the thermodynamic force between substrates $k$ and products $j$ that will lead to flows of matter via reactions from reactants to products and vice versa until the equilibrium or non-equilibrium stationary state $\nabla \mu_{j}=0$ is attained. When an electromagnetic field, e.g., quanta $\Delta Q_{j k}$ of sunlight, couple to a reaction between $k$ and $j$, it will generate a potential difference $\sum \mu_{k}+\Delta Q_{j k}-\mu_{j}$, abbreviated here by $\nabla \mu_{j}$, that forces a chemical reaction, i.e., an energy flow toward non-equilibrium concentrations $N_{k}$ and $N_{j}$. Likewise, when an electric potential gradient $\nabla \Phi_{E}$, i.e., an electric field couples to a charged entity $j$, it will lead to ionic flows and electric currents. Thus, the concept of an external field merely serves to denote energy densities that are not described explicitly by sources. For example, according to Gauss's law the electric field gives the net effect of some charges that are not explicitly specified whereas those charges, that are explicitly described, are regarded as integral parts of the system. The subjective choice to label some energy densities as entities of the (local) system and to refer to others as the (global) surroundings is unimportant. In any case it is the differences in energy within the system and with respect to its surroundings that power evolution. The advantage of Gibbs formalism is that any differences between diverse energy densities $\Phi_{j}$ inflict forces, whereas the specific nature of each $\Phi_{j}$ is immaterial. Owing to the transforming flows of energy the internal just as the external densities-in-energy keep changing during the energy transduction. Consequently, the forces of evolution keep changing and the system keeps redirecting its course of evolution. Without invariants of motion trajectories of non-conserved systems are non-deterministic and unpredictable in details.

Evolution as dispersal of energy is described by the overall rate of change in entropy (Sharma and Annila, 2007)

$\frac{\mathrm{d} S}{\mathrm{~d} t}=R \sum_{j=1} \frac{\mathrm{d} N_{j}}{\mathrm{~d} t} \nabla \frac{\mu_{j}}{R T}=R \sum_{j=1} D_{j}\left(\frac{\mu_{j}}{R T}\right)$

which is the equation of motion for evolution. The grand sum of Eq. (1) over all constituents $j$, i.e., repositories of energy defines the system as an energy transduction network (Fig. 1). When the network evolves by flows of energy to attain a stationary state in its high-energy surroundings, it may emerge with or acquire increasingly more effective mechanisms of energy transduction. For example, on Earth evolution over the eons has resulted in a contemporary cell that is a highly effective energy transduction system where metabolic pathways connect chemical potentials of diverse metabolites with each other and with the surrounding energy density. According to the universal 2nd law, the objective of energy dispersal is expected to be the same in other circumstances and locations but the courses of evolution and its mechanistic manifestations are anticipated to differ in details. Likewise an ecosystem is a biotic transduction network, commonly known as a food chain that has evolved to transform high-energy sunlight to thermal radiation. In general, thermodynamic systems evolve by consuming free energy, i.e. potential energy differences and gradients, using those transduction mechanisms that they happen to posses at a given

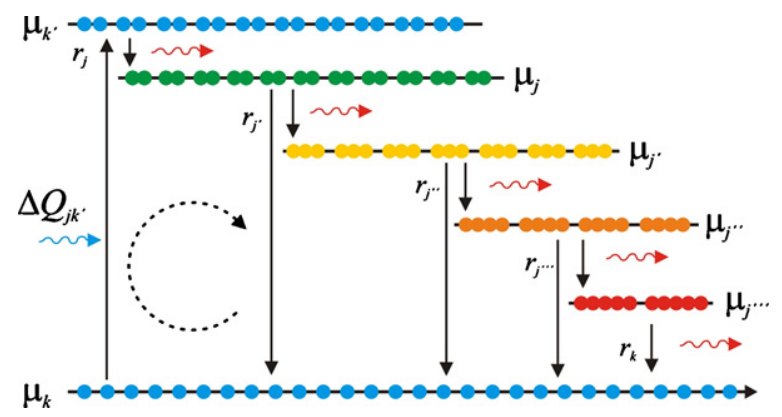

Fig. 1. Energy level diagram of a transduction network represents an open system. All flows of energy (arrows) take the direction down along thermodynamic gradients $\nabla \mu_{j}$. This will result in diverse transformation and transport processes. When external energy (blue wavy arrow) $\Delta Q_{j k}$ couples to a $j k$-reaction (black arrows) it will add to the substrate $\mu_{k}$ to result in a transition state $\mu_{k \text { r }}$ that will relax to the product $\mu_{j}$. Consequently the products appear in high-energy non-equilibrium concentrations $N_{j}$. The high chemical potential $\mu_{j}$ may in turn fuel by coupled reactions the entire dissipative (red wavy arrows) chain of energy transduction $j \rightarrow j^{\prime} \rightarrow j^{\prime \prime} \rightarrow j^{\prime \prime \prime}$ toward the equilibrium. This generates incessant circulation of matter (dashed circle) which transforms the high-energy influx to low-energy efflux. The overall transduction rate increases when the mechanism-associated rate coefficients $r_{j}$ increase, e.g., due to emerging catalyses. Integrated catalysis along the chain of transduction will increase the overall rate of dispersal from high-energy sources to low-energy sinks. This quest to disperse energy more and more effectively will give rise to hierarchical systems, e.g. ecosystems. However, when particular basic constituents that couple to the external energy spectrum are scarce, evolution will stall already at low thermodynamic levels of hierarchy where only elementary processes such as diffusion, heat flows and simple chemical reactions generate dissipation. 
time. When various systems draw from common pools of material constituents and energy, those systems equipped with most effective mechanisms conduct the largest flows whereas those with least effective means may acquire hardly anything. The general thermodynamic description of interacting transduction systems is phrased in biological terms as the struggle for existence.

It is important to realize that the gradients, in particular the surrounding fields, impose the direction of evolution on an open system. When high-energy surroundings couples to the transformation processes, the system evolves by increasing its energy content by influx toward the non-equilibrium stationary state (Fig. 1). Conversely, when the system is high in energy, it evolves toward equilibrium by decreasing its energy content by efflux to its low-energy surroundings. This extends the familiar Le Châtelier's principle (Atkins, 1998), i.e. the chemical equilibrium depends on conditions, to evolution whose direction depends on the surrounding densities-in-energy. The directionality in energy transduction is mathematically expressed by Eq. (1) via directional derivates $D_{j}$ that define a time-dependent affine manifold of energy densities by tangent vectors (Lee, 2003; Kaila and Annila, 2008).

Forces due to internal and surrounding thermodynamic gradients are consumed in energy transduction processes. Each of the resulting flows is proportional to the specific gradient (Sharma and Annila, 2007)

$v_{j}=r_{j} \nabla \frac{\mu_{j}}{R T}$

by a mechanistic rate constant $r_{j}>0$ to satisfy the balance $v_{j}=-\sum v_{k}$ in the transformations from substrates $N_{k}$ to products $N_{j}$. The change in momentum is balanced by the change in mass which amounts from changes in interaction energies that distinguish the identity $k$ from $j$ (Kaila and Annila, 2008). The transformationassociated dissipation is apparent from chemical reactions that are either endergonic or exergonic. To acknowledge the dissipation in transformations is conceptually very important, although the mass change corresponding to the energy influx or efflux due to electronic restructuring in chemical bonding is small, e.g., compared with the mass change in transformations that involve atomic nuclei. As long as the energy gradient $\nabla\left(\mu_{j} / R T\right)>0$, the population $N_{j}$ may increase by transport and transformation processes at the expense of other ingredients $N_{k}$. For example, an animal population may grow as long as there is food for it. On the other hand when $\nabla\left(\mu_{j} / R T\right)<0$, the population $N_{j}$ will face cuts one way or another for the benefit of other populations $N_{k}$ in the system or in its surroundings.

When Eqs. (1) and (2) are combined to

$$
\frac{\mathrm{d} S}{\mathrm{~d} t}=R \sum_{j=1} r_{j}\left(\nabla \frac{\mu_{j}}{R T}\right)^{2} \geq 0
$$

it is seen from the quadratic form that entropy is indeed increasing in all processes. Life is no exemption. The principle of increasing entropy is equivalent to the principle of decreasing free energy $\mathrm{d} G / \mathrm{d} t \leq 0$ by the definition $G=H-T S$ where $S$ is entropy, and enthalphy $H=\sum U_{j}+R T \sum N_{j}$ containing internal energy $U_{j}$ as usual. It is emphasized that the system evolves by acquiring or expelling energy, hence the thermodynamic quantities also change during evolution.

An open system will generate more and more power when consuming the external potential energy. The free energy is used to make energy transduction mechanisms and to maintain the populations when the stationary state has been reached. The condition $\mathrm{d} S / \mathrm{d} t=\mathrm{d} G / \mathrm{d} t=0$, familiar from chemical equilibrium (Atkins, 1998), determines the stationary populations $N_{j}$ of transduction mechanisms $j$, i.e. distributions of densities with characteristic skew
(Grönholm and Annila, 2007; Würtz and Annila, in press). These distributions from photon radiation law (Planck, 1901) to the distribution of galaxies (Choloniewski, 1985) as well as from gene lengths (Skovgaard et al., 2001) to animal populations (Rosenzweig, 1995) are closely approximated by log-normal distributions (Kapteyn, 1903; Limpert et al., 2001). In contrast, those distributions that are independent from energy, such as a phase-angle distribution, are symmetric.

At the stationary state driving forces balance each other and net flows of energy vanish. However the stationary state corresponding to the maximum $S=R \sum N_{j}$ is not without the motions that maintain the populations $N_{j}$. For example, a cell maintains a constant metabolic flux when furnished with a steady supply of food. Likewise, an engine is running at a constant rate when fueled by a steady flow of external energy. These steady-state conserved motions generate power when maintaining the vital flows and replenishing mechanisms. At this stationary state the total potential energy $U$ and influx $Q$ equate with the total kinetic energy $K$ that manifests as maximal flows. Therefore, at the maximum entropy state the power that is obtained in analogy to an electric circuit from Eqs. (1) and (2) in terms of the flows (currents) is at the maximum (Lotka, 1922). During evolution toward increasingly more effective energy transduction the net energy influx $\mathrm{d} Q / \mathrm{d} t$ is deposited at increasingly larger potentials $U$ and generating correspondingly more motions, in terms of kinetic energy $K$, according to the balance equation $\mathrm{d} U / \mathrm{d} t+\mathrm{d}(2 K) / \mathrm{d} t=\mathrm{d} Q / \mathrm{d} t$ (Kaila and Annila, 2008).

Importantly, the thermodynamic imperative to level differences in energy densities does not encourage simple squandering. Instead, when free energy is used to assemble new mechanisms, new potentials may be accessed by them to propel evolution further. Since no single entity, due to its finite composition, may house all conceivable characteristics to tap into all conceivable potentials, mechanistic differentiation, e.g. niche differentiation is encouraged (Rosenzweig, 1995). Also standardization will enhance mutual interactions (Jaakkola et al., 2008a) that level differences in energy densities more and more effectively. Thus, organizations are regarded, according to the 2nd law, as machinery in order to disperse energy.

\section{The criterion of natural selection}

When several mechanisms are available, the energy flows will distribute among them to achieve the highest throughput in accordance with the maximum entropy production principle and the maximum power principle. It follows from this that energy will flow naturally along the steepest descents in the energy landscape, equivalent to the steepest ascents on the entropy manifold (Kaila and Annila, 2008; Sharma and Annila, 2007) to abolish differences most rapidly. In this way the overall probability $P$, related to the entropy increase by $\mathrm{d} S / \mathrm{d} t=R d(\ln P) / \mathrm{d} t$, will increase most rapidly. Thus, the mechanistic rate constants $r_{j}$ in Eqs. (2) and (3) are of particular interest as they will affect the rate of energy dispersal, the speed of leveling gradients. Especially, when systems acquire free energy (Morowitz, 1968) from common surrounding sources and fields, those system with most effective mechanisms will catch most of the flow (Matsuno and Swenson, 1999). This is often referred to as the struggle for existence. The competition is incessant among systems that tap into a common high-energy source. Their mechanisms of energy transduction are regenerated from basic stable constituents over and over again. For example, various enzymes catalyzing various metabolic pathways can be seen to compete for common metabolites just as animals are viewed to compete for a common food stock. Scarcity of resources generates the bias for the emergence of increasingly more effective mechanisms. For a given gradient any increase in the mechanis- 
tic rate $r_{j}$ will speed up evolution. Therefore there is a functional bias for increased rates $r_{j}$ to channel energy more rapidly. For example, although carbon dioxide does dissociate spontaneously in water, evolution has emerged with carbonic anhydrase, an enzyme with an amazing turnover about one million molecules in a second.

Any mechanism of energy transduction is according to Eq. (3) a result of flows. Due to random variation in flows, e.g. due to sporadic fluctuations in energy densities, a new mechanism may assemble. It may tap into new potentials and generate new flows. Those mechanisms that provide rapid increase of entropy are naturally selected and those that channel less are discarded. Thus it has been argued that the rate of entropy increase $\mathrm{d} S / \mathrm{d} t>0$ is the universal fitness criterion of natural selection (Sharma and Annila, 2007). It is equivalent to the rate of free energy decrease $\mathrm{d} G / \mathrm{d} t<0$. In other words, systems are driven for growth to consume all available surrounding thermodynamic gradients by increasing more effective and diverse mechanisms. Initially superfluous supplies may be dispersed through many mechanisms, but when the resources reduce, flows funnel along the steepest gradients in the free energy landscape, i.e., via the most effective mechanisms. Thus the initial disparity among various mechanisms will narrow to the final diversity of highly effective mechanisms (Gould, 1991). By the same token integration to hierarchical organization is regarded as a mechanism to disperse energy. The natural selection is not limited to particular mechanisms of variation such as genetic mutations but acts on all matter. Therefore it is concluded that the law for energy dispersal with the greatest dissipation rates underlies the emergence of functional structures, regularity and hierarchical organization.

Although the imperative to reduce gradients is simple, the course of evolution can be intricate. For example, highly effective mechanisms may temporarily exhaust their potentials. Wellknown examples are oscillating Belousov-Zhabotinsky chemical reactions, prey-predator populations as well as economical fluctuations. Conversely when resources, i.e., surrounding fields begin to diminish, all systems are forced to decline and disintegrate. Then it is the high internal potential energy $U$ of the system that accumulated during the growth phase which turns to fuel its external surroundings that are lower in energy density. In such a 'plight' a system may also turn to 'self-cannibalism' or autophagy by internal exclusion. For example, when a eukaryotic cell is deprived of metabolites, it turns to digest its own cytoplasm (Yorimitsu and Klionsky, 2005). Furthermore, the growth is rarely steady but displays punctuations and stasis. Every time when a new mechanism emerges, the rate of energy transduction increases rapidly, i.e. punctuates, and when potential energy differences are about to vanish, the process will level to the stationary state stasis. In particular, larger systems grow in a piecewise sigmoid manner. The intermittent course of punctuations and stases is characteristic of biotic just as abiotic evolution (Eldredge and Gould, 1972; Sneppen et al., 1995). The overall sigmoid course of a natural process (Eq. (2)) is non-deterministic but often closely approximated by the deterministic logistic equation (Strogatz, 2000; Verhulst, 1845). Initially the thermodynamic gradient is large but the rate of evolution is limited by the newly emerged mechanistic capacity. When more mechanisms emerge, the flow of energy increases rapidly but later it slows down toward the stasis, being then limited by the diminishing gradient. Animate just as inanimate growth phenomena following Eq. (2) display the characteristic sigmoid course that is mostly a straight line on a $\log -\log$ plot, i.e. a power-law. The cumulative curve is the familiar diversity relationship between the number of species and sampling area (Rosenzweig, 1995; Würtz and Annila, in press).

\section{Evolution to hierarchy}

The holistic and scale-independent description of energy transductions systems, provided by the Eqs. (1)-(3), regards each entity as a system of its own. For example, cells of an organism are energy transduction systems of their own; just as their constituents, molecules are systems of atoms and so on. Each entity is viewed as an energy transduction system that has resulted from an earlier natural process. For example, elements that emerged from a primeval dissipative stellar synthesis are now constituents of biotic transduction mechanisms on Earth. Over the eons the global system has evolved to house a large diversity of mechanisms, in particular those that we refer to as species in the quest for increased energy transduction from the hot Sun to the cold space (Brooks and Wiley, 1986; Chaisson, 2001; Karnani and Annila, 2008; Lovelock, 1988; Schneider and Kay, 1994; Weber et al., 1989). Today, just as earlier, variation in syntheses of mechanisms may yield even more effective mechanisms. Furthermore, to maintain high transduction, rapid restorations of mechanisms are favored. Contemporary biotic and economic mechanisms are rapidly regenerated by informationguided syntheses to maintain high rates of energy transduction.

According to thermodynamics an entity, e.g., an atom, molecule, cell, organism, ecosystem, economy, etc. is regarded merely as an energy transduction system. Obviously the amount of energy that is dissipated, e.g., by a single hydrogen bond formation in a molecular system is minute in comparison to the overall energy transduction of an entire ecosystem. The scale is different-the principle is the same. Diverse systems are only valued by their rates of entropy increase, i.e., abilities to conduct energy. Thus, the self-organization to a hierarchical system is warranted only when it will provide rates that are superior to its submerged systems operating as independent systems. A large integrated system will appear when the total energy flow via the sum $\sum_{j}$ over its functional entities with rate constants $r_{j}$ in Eq. (3) will be larger than that produced by its components as individuals. Likewise, a large organization will be bound for disintegration when conditions change so that a higher overall rate $\mathrm{d} S / \mathrm{d} t$ will be provided by its constituents as independent operators. In other words, the rise and fall of hierarchies is governed by the same ubiquitous law of energy dispersal.

When examining diverse systems at various levels of hierarchy, it is apparent that systems tend to accommodate diverse subsystems, usually not only of one kind. For example, a eukaryotic cell houses various endomembranes and organelles. An organism contains different kinds of organs. Highly developed economies are also widely dispersed and specialized in their machinery and activities. Diversity within diversity can be understood from the thermodynamic imperative. Characteristics of atoms, properties of molecules, functions of cells, phenotypes of organisms and structures of economies are only valued according to their contribution to decrease free energy or equivalently to increase entropy (Brooks and Wiley, 1986; Farrell and Winkler, 2006; Georgescu-Roegen, 1971; Schneider and Kay, 1994). Customarily, when using theory of non-equilibrium thermodynamics, a distinction is made between local and global changes in entropy. However, when the entropy increase is defined via Eq. (1) as the free energy decrease, it is immaterial how one chooses to partition nature to a system and its surroundings. When gradients are leveling, both entropy of the system and its surroundings will increase. It is a mere consequence of the total conservation of energy in the flows that cross the mutual border between the system and surroundings. The holistic and self-similar formalism is particularly useful when describing hierarchical systems where the surroundings of a system at each level of organization is a larger system itself. When different kinds of entities interact with each other, they form an integrated system capable of higher dispersal of energy than a system composed of 
homogenous constituents all performing the same function without mutual benefits. The mutual interactions, e.g. chemical bonds, interactions among molecules, services between endosymbiotic cellular organelles (Margulis, 1976) and between species in an ecosystem just as actions between players on markets, bind the entities together to a system capable of increased dissipation. 'Stoichiometry' of entities, i.e. their numbers $N_{j}$ in a system, is governed by $\mathrm{d} S / \mathrm{d} t=\sum\left(\mathrm{d} S / \mathrm{d} N_{j}\right)\left(\mathrm{d} N_{j} / \mathrm{d} t\right)>0$. The universal criterion allows to evaluate whether the rate of free energy decrease could possibly increase by including one more constituent $N_{j}+1$ of an existing kind $j$ or by including a new kind of a constituent $j+1$ to the system. At the level of an ecosystem this is the condition of speciation. Likewise, in economical systems producers aim to tailor their portfolios of goods on the same basis to supply optimally the demand. The choice is tricky, more precisely non-deterministic, because a change in one flow will affect other flows.

In general, mechanisms of particular transduction machinery are revealed to an observer by the system's dispersion relation, i.e., by the energy response function. Characteristic energies of dissipation, for example, absorption bands of a molecular system, e.g., are exposed by a scan over a frequency range. Recorded lineshapes report from forms of potentials. Likewise, a spectrum of species in an ecosystem displays dispersion of chemical energy. Population distributions report from shapes of free energy basins, customarily known as ecological niches. Similarly an economy will respond by its characteristic mechanisms to fluxes of goods and capital that are forms of energy too. According to Eqs. (1) and (3), a large and sophisticated system covers by its many and versatile mechanisms, denoted by the sum over $j$, a wide dispersion of energy with high capacity. Thus it is able to diminish diverse gradients effectively. Furthermore, a versatile system with its many mechanisms is also capable of redirecting its flows in response to external changes. Customarily this is referred to as adaptation. An organism, an ecosystem or a society that is highly developed in its energy transduction has obviously more to lose in a catastrophe but also many more means to recover from it than a less developed system. Diversity of mechanisms contributes to the stability of the system. When the sum in Eq. (1) extends over numerous constituents $j$ and contains numerous transformation $j k$-paths, the transduction system is stable against perturbations. The many repositories of energy hold capacity to resist changes and the many mechanisms provide means to redirect flows in order to adapt to the changes. For example, the particular mechanisms that account for the robustness of cellular function are being unraveled (Stelling et al., 2004). External just as internal long-term stability gives opportunities for mechanisms to emerge and to level even small potential energy differences, i.e., to operate on small margins. On the contrary, when supplies are rapidly fluctuating effective and integrated systems will not rise. In this case, the returns measured by $\mathrm{d} S / \mathrm{d} t$ do not encourage investments in mechanisms that would soon, when the conditions change, become obsolete, i.e., too high in energy relative to the impoverished surroundings. Thus it is concluded that the incentive $\mathrm{d} S>0$ is the general condition of integration just as it is for disintegration.

All integration processes impose new surroundings on the submerged systems. Consequently restructuring of the internal processes is inherent in the integration processes and in fact necessary to obtain the increased rates of dispersal, customarily known as gains in synergy. For example, the highly integrated processes among eukaryote organelles reflect their long evolutionary history together, just as the division of labor among differentiated cells of an organism. Likewise, national economies are presently in for restructuring for increased efficacy in transactions when integrating into the global economy. Apparently the global system is special at least in our solar system to house a suitable mix of substances, most notably ingredients of carbon chemistry, to couple to the highenergy flux from Sun, as well as with other raw materials that can be industrially processed by other intensive sources of energy. Integration into increasingly larger nested organizations is, according to thermodynamics of open systems, only a more effective means to diminish potential differences.

An organization will disassemble when it fails to generate a larger $\mathrm{d} S / \mathrm{d} t$ rate than its submerged systems. Hierarchies will fall in response to decreasing surrounding supplies and internal mechanistic failures. Any system is bound for destruction when it is deprived from surrounding supplies and associated flows. Accordingly, senescence of dissipative structures is inevitable (Salthe, 1989). When the internal energy density of the system is higher than that of surroundings, flows of energy will direct from the system to its surroundings. Obviously an animal will starve to death when deprived from food and its carcass will be a supply for other surroundings systems. A pathogen when marked for isolation from its supplies by antibodies, will struggle to survive in isolation. Likewise, economic and social systems may impose embargos on each other in hopes to alter on-going internal processes.

\section{Boundaries between systems}

Mathematical concepts of non-linear dynamics (May, 1976; Strogatz, 2000) are most convenient to describe the energy landscape that is flattening during evolution (Fig. 2). The manifold of 'basins within basins' corresponds to 'systems within systems'. For example, molecules adhere to one another by numerous weak interactions to make a cell corresponding to a larger basin. Cells attach to each other to make an organ corresponding to an even larger

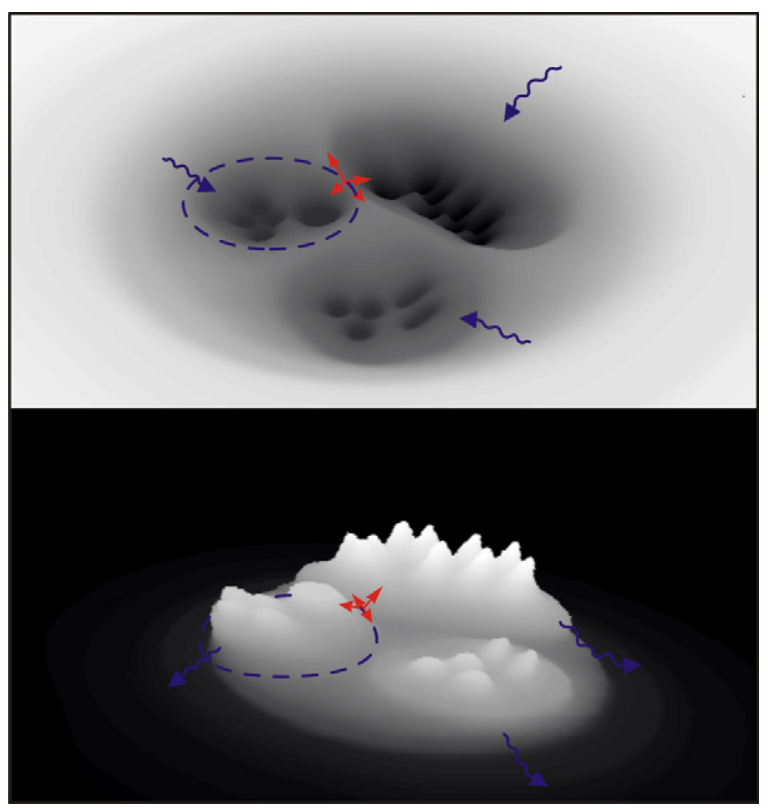

Fig. 2. Free energy landscape is depicted to house a hierarchy of energy transduction systems that evolve toward a non-equilibrium stationary state (above) or toward equilibrium (below) with respect to the surrounding density-in-energy. When the surrounding density-in-energy is high (light), the systems are basins (dark) in the landscape. These sites have mechanisms to absorb energy (blue wavy arrows). Each basin of attraction (habitant) is encircled by separatrices that mark the boundaries of the system (exemplified by a blue dashed circle). A set of smaller systems organize for increased dissipation to a larger system that localizes to a larger and deeper basin. The submerged smaller wells connect via saddle points (exemplified by red crossed arrows) that channel the strongest mutual integrating interactions. On the other hand when the surrounding density-in-energy is low (dark), the high-energy systems are flattening heights (light) in the landscape. These sites have mechanisms to dissipate their internal energy in the quest for equilibrium. 
basin and organs assemble to an individual corresponding to yet a larger basin. Stationary system are stable against population fluctuations $\delta N_{j}$ according to the Lyapunov stability criterion (Kondepudi and Prigogine, 1998; Strogatz, 2000) $\mathrm{d} G\left(\delta N_{j}\right)>0, \mathrm{~d} G\left(\delta N_{j}\right) / \mathrm{d} t<0$. Any internal perturbation $\delta N_{j}>0(<0)$ away from the minimum free energy will give rise to restoring driving forces $\nabla\left(\mu_{j} / R T\right)<0(>0)$. The corresponding basin of attraction is encircled by separatrices that mark the boundary of a system. The border is the ridge in free energy that surrounds the basin. System dynamics is usually disclosed by linearization of the basin's shape to give Jacobian whose trace, determinant and discriminant reveal eigenmodes, the characteristic motional modes (Strogatz, 2000). These modes may change when the landscape of a non-conservative system evolves due to influxes and effluxes.

The border of an evolving system is changing. For example, when an atom moves from one molecule to another via a chemical reaction, the forms of two molecular free energy basins will change in the process. Likewise, a population change will affect the available supplies, i.e. the shape of a basin referred to as a niche. Obviously a common border cannot be moved without affecting both neighbors. In other words, nature is integrated by mutual interactions so that the ceteris paribus principle does not hold (Gould, 2002). When two systems with very similar mechanisms of energy transduction compete for the same resources the bordering ridge in between will vanish in a merger. Alternatively, the one of the two systems with slightly inferior mechanisms will be eventually excluded by the more potent one (Gause, 1934). The precise evolutionary course depends on numerous factors but the overall direction is a mere consequence of energy dispersal.

The definition of a border is somewhat of a subjective decision. However, energy transduction systems naturally structure themselves depending on strengths and ranges of interactions. In general, interactions are effectively weaker over increasingly longer ranges. The strongest forces adhere to the smallest systems, which have the fastest stationary motions. For example, chemical reactions in a cell are faster than intercellular trafficking in an organism, which is still faster than matter flows in an ecosystem. Small systems sustain, via strong mutual interactions, intense communication among their entities. The decreasing densities-in-energy go hand in hand with increasingly larger systems. In other words, the curvature of an energy-density landscape decreases with increasing length scale. This is the reason for the characteristic asymmetry about the boundary between a larger system and its submerged smaller systems. The larger system is not merely a scaled copy of a subsystem but differs by its interactions that contribute beyond the mechanisms of subsystems to energy dispersal. Stratified structures, i.e., distinct boundaries, are there where strengths and ranges of forces change substantially. These are the places for natural borders.

\section{Discussion}

The view of nature as a hierarchically nested energy transfer system, provided by the equation of motion (Eq. (1)), may at first appear as a highly simplified description of complex organization. The austere quest to abolish energy gradients may appear utterly insufficient to describe intricacies of evolution. However, despite the concise notation, the equation of evolution contains every entity of matter and every quantum of energy. The general formalism is not approximate but abstract. For example, the Gibbs free energy and population densities can be readily obtained for simple chemical systems. For an ecosystem it would, of course, be an overwhelming effort to catalog everything in such details. Nevertheless, even without the precise knowledge of numbers, the statistical mechanics formalism gives understanding by keeping track of all energy densities and flows of energy between them. Evolutionary courses of large systems can be intricate and the mechanisms of dissipation can be sophisticated but these characteristics should not obscure that the underlying principle of increasing entropy is universal. In this sense the present analysis is not unique among many earlier studies that have rationalized diverse evolutionary phenomena using the 2nd law of thermodynamics that is often expressed in terms of increasing entropy rather than in terms of energy dispersal. The correspondence according to Eq. (1) between decreasing free energy and increasing entropy is valuable because in some cases it is easier to perceive decreasing potential energy differences and in other cases increasing energy flows.

Energy transduction systems integrate into larger systems for increased dissipation because no system can become larger than the one where its entities still reach to interact with each other. Without interactions there is no system. For example, molecules may take part only in reactions with substrates that they can diffuse to within their lifetimes. A cell by its increased range of interactions, e.g. by motility, is capable of further dissipation by having an access to a larger pool of potentials. Therefore systems structure to functional hierarchical organizations according to interaction strengths and ranges. The surroundings affect not only via exchange processes but impose forces on system's internal processes. These dualistic characteristics and their consequences have been addressed extensively earlier (Brooks, 2000; Maynard Smith and Szathmary, 1999). The increased rate of energy transduction as the natural selection criterion is particularly commanding when the system finds no means to expand or may even have to retract due to curtailed supplies. Restructuring of energy transduction processes within the system in response to the changes in gradients may even result in superior mechanisms to access new potentials and to generate growth above that preceding the crisis (Harris, 1979).

Although evolution is unambiguously on its way to the grand stationary state devoid of gradients, the natural process is nondeterministic in its details because the flows affect the driving forces that in turn direct the flows. In other words, the free energy landscape unfolds concurrently with evolution and prevents from predicting future in detail. Evolution can also be chaotic as in the familiar case of the three-body problem (Sundman, 1912). When initial densities-in-energy vary substantially, trajectories of evolution are complicated but gradually the highly crumpled nonEucledian landscape is flattening via numerous dissipative flows toward the cold space. The objective is not to reduce biology to deterministic physics but to amend the formalism of physics with the holistic characteristic of biology.

The consilience due to the 2nd law of thermodynamics is all inclusive. All processes, animate just as inanimate, disperse energy (Schneider and Sagan, 2005) in accordance with the famous quote from Eddington (Eddington, 1927) that "The law that entropy always increases - the second law of thermodynamics - holds, I think, the supreme position among the laws of Nature." Over the eons the dissipative structures (Glansdorff and Prigogine, 1971), i.e. open non-equilibrium systems on Earth have evolved for increased energy transduction by integrating into the hierarchical global ecosystem in response to the immense stream of solar energy. Today the integration of national economies into the global economic system follows the same principle but is extensively powered by non-renewable energy. Now these resources are diminishing, and if not substituted by others, the global economy will face inevitable disassembly (Diamod, 2005). Far further in time and beyond the needs of mankind also the solar supplies will be exhausted. Still further in future it is expected that the remains of the solar system will be dissipated by an evaporating black hole (Hawking, 1974) in the galactic churn. 


\section{Acknowledgments}

We thank Mahesh Karnani for enlightening discussions and valuable comments.

\section{References}

Annila, A., Annila, E., 2008. Why did life emerge? Int. J. Astrobiol. 7, 293-300.

Atkins, P.W., 1998. Physical Chemistry. Oxford University Press, New York.

Boltzmann, L., 1886. The second law of thermodynamics. In: McGinnes, B. (Ed.), Ludwig Boltzmann, Theoretical Physics and Philosophical Problems. Reidel, p. 1974.

Brooks, D.R., Wiley, E.O., 1986. Evolution as Entropy: Toward A Unified Theory of Biology, 2nd ed. The University of Chicago Press, Chicago.

Brooks, D.R., 2000. The nature of the organism: life takes on a life of its own. Proc. N.Y. Acad. Sci. 901, 257-265.

Carnot, S., 1824. Reflexions sur la puissance motrice du feu sur les machines propres a developper cette puissance. Paris. Mendoza, E. (Ed.) 1960. Reflections on the motive power of fire-and other papers on the second law of thermodynamics by E. Clapeyron and R. Clausius. Dover Publications, New York.

Chaisson, E.J., 2001. Cosmic Evolution: The Rise of Complexity in Nature. Harvard University Press, Cambridge.

Clausius, R., 1879. Mechanical Theory of Heat, 2nd ed. Macmillan \& Co., London.

Choloniewski, J., 1985. Bivariate luminosity function of E-galaxies and SO-galaxies. Mon. Not. R. Astron. Soc. 214, 197-202.

Darwin, C., 1859. On the Origin of Species. John Murray, London.

Dewar, R., 2003. Information theory explanation of the fluctuation theorem, maximum entropy production and self-organized criticality in non-equilibrium stationary states. J. Phys. A: Math. Gen. 36, 631-641.

Diamod, J., 2005. Collapse: How Societies Choose to Fail or Succeed. Viking Books, New York.

Eddington, A.S., 1927. The Nature of the Physical World (Gifford Lectures), Macmillan reissued (1995). AMS Press.

Eldredge, N., Gould, S.J., 1972. In: Schopf, T.J.M. (Ed.), Models in Paleobiology. Freeman Cooper, San Francisco, pp. 82-115.

Farrell, K.N., Winkler, R., 2006. The new entropy law and the economic process. Ecol. Complex. 3, 265-274.

Gause, G.F., 1934. The Struggle for Existence. Williams \& Wilkins, Baltimore, MD.

Georgescu-Roegen, N., 1971. The Entropy Law and the Economic Process. Harvard University Press, Distributed by Oxford University Press, London.

Gibbs, J.W., 1876. On the equilibrium of heterogeneous substances. Trans. Conn. Acad. Arts Sci. 3, 108-248; Gibbs. J.W., 1878. On the equilibrium of heterogeneous substances. 3, 343-524; Gibbs, J.W., 1993-1994. The Scientific Papers of J. Willard Gibbs. Ox Bow, Woodbridge CT.

Glansdorff, P., Prigogine, I., 1971. Thermodynamic Theory of Structure, Stability, and Fluctuations. Wiley-Interscience, New York

Gould, S.J., 1991. The disparity of the Burgess shale arthropod fauna and the limits of cladistic analysis: why we must strive to quantify morphospace. Paleobiology $17,411-423$.

Gould, S.J., 2002. The Structure of Evolutionary Theory. Harvard University Press, Cambridge, MA.

Grönholm, T., Annila, A., 2007. Natural distribution. Math. Biosci. 210, 659-667.

Gyarmati, I., 1970. Nonequilibrium Thermodynamics: Field Theory and Variational Principles. Springer-Verlag, New York.

Harris, M., 1979. Cultural Materialism-The Struggle for a Science of Culture. Random House, New York.

Hawking, S.W., 1974. Black hole explosions. Nature 248, 30-31.

Jaakkola, S., Sharma, V., Annila, A., 2008a. Cause of chirality consensus. Curr. Chem. Biol. 2, 53-58.

Jaakkola, S., El-Showk, S., Annila, A., 2008b. The driving force behind genomic diversity. Biophys. Chem. 134, 232-238, arXiv:0807.0892.

Jaynes, E.T., 1957. Information theory and statistical mechanics. Phys. Rev. 106, 620-630.

Kaila, V.R.I., Annila, A., 2008. Natural selection for least action. Proc. R. Soc. A 464, 3055-3070.

Kapteyn, J.C., 1903. Skew Frequency Curves in Biology and Statistics. Astronomical Laboratory, Noordhoff, Groningen.
Karnani, M., Annila, A., 2009. Gaia again. BioSystems 95, 82-87.

Kondepudi, D., Prigogine, I., 1998. Modern Thermodynamics. Wiley, New York.

Lee, J.M., 2003. Introduction to Smooth Manifolds. Springer-Verlag, New York.

Limpert, E., Stahel, W.A., Abbt, M., 2001. Log-normal distributions across the sciences: keys and clues. Bioscience 51, 341-352.

Lorenz, R.D., 2002. Planets, life and the production of entropy. Int. J. Astrobiol. 1, 3-13.

Lotka, A.J., 1922. Natural selection as a physical principle. Proc. Natl. Acad. Sci. U.S.A. $8,151-154$.

Lovelock, J.E., 1988. The Ages of Gaia. Oxford University Press, London.

Lucia, U., 1995. Mathematical consequences and Gyarmati's principle in rational thermodynamics. Il Nuovo Cimento B110, 1227-1235.

Lucia, U., 2008. Probability, ergodicity, irreversibility and dynamical systems. Proc. R. Soc. A 464, 1089-1104.

Margulis, L., 1976. Genetic and evolutionary consequences of symbiosis. Exp. Parasitol. 39, 277-349.

Maupertuis, P.L., 1746. Les Loix du mouvement et du repos déduites d'un principe metaphysique. Histoire de l'Académie Royale des Sciences et des Belles Lettres, 267-294.

Matsuno, K., Swenson, R, 1999. Thermodynamics in the present progressive mode and its role in the context of the origin of life. BioSystems 51, 53-61.

Matsuno, K., Salthe, S.N., 2002. The origin and development of time. Int. J. Gen. Syst. 31, 337-393.

May, R.M., 1976. Simple mathematical models with very complicated dynamics. Nature 261, 459-467.

Maynard Smith, J., Szathmary, E., 1999. The Origins of Life. Oxford Univ. Press, Oxford.

Morowitz, H., 1968. Energy Flow in Biology. Academic Press, New York.

Planck, M., 1901. On the law of distribution of energy in the normal spectrum. Annalen der Physik 4, 553-563.

Rosenzweig, M.L., 1995. Species Diversity in Space and Time. Cambridge University Press, Cambridge.

Salthe, S.N., 1985. Evolving Hierarchical System. Columbia University Press, New York.

Salthe, S.N., 1989. Self-organization of/in hierarchically structured systems. Syst. Res. 6, 199-208.

Salthe, S.N., 1993. Development and evolution: Complexity and Change in Biology. MIT Press, Cambridge, MA.

Salthe, S.N., 2004. The spontaneous origin of new levels in a scalar hierarchy. Entropy 6, 327-343.

Schneider, E.D., Kay, J.J., 1994. Life as a manifestation of the second law of thermodynamics. Math. Comp. Model. 19, 25-48.

Schneider, E.D., Sagan, D., 2005. Into the Cool: Energy Flow, Thermodynamics, and Life. The University of Chicago, Press, Chicago.

Shannon, C.E., 1948. A mathematical theory of communication. Bell Syst. Tech. J. 27, $379-423,623-656$

Sharma, V., Annila, A., 2007. Natural process-natural selection. Biophys. Chem. 127, 123-128.

Skovgaard, M., Jensen, L.J., Brunak, S., Ussery, D., Krogh, A., 2001. On the total number of genes and their length distribution in complete microbial genomes. Trends Genet. 17, 425-428.

Sneppen, K., Bak, P., Flyvbjerg, H., Jensen, M.H., 1995. Evolution as a self-organized critical phenomenon. Proc. Natl. Acad. Sci. U.S.A. 92, 5209-5213.

Stelling, J., Sauer, U., Szallasi, Z., Doyle, F., Doyle, J., 2004. Robustness of cellular functions. Cell 118, 675-685.

Strogatz, S.H., 2000. Nonlinear Dynamics and Chaos with Applications to Physics, Biology, Chemistry and Engineering. Westview, Cambridge, MA.

Sundman, K.E., 1912. Memoire sur le probleme de trois corps. Acta Math. 36, 105179.

Ulanowicz, R.E., Hannon, B.M., 1987. Life and the production of entropy. Proc. R. Soc. B 232, 181-192.

Yorimitsu, T., Klionsky, D.J., 2005. Autophagy: molecular machinery for self-eating. Cell Death Differ. 12, 1542-1552.

Verhulst, P.F., 1845. Recherches mathématiques sur la loi d'accroissement de la population. Nouv. Mém. Acad. R. Sci. Belleslett. Bruxelles 18, 1-38.

Weber, B.H., Depew, D.J., Dyke, C., Salthe, S.N., Schneider, E.D., Ulanowicz, R.E., Wicken, J.S., 1989. Evolution in thermodynamic perspective: an ecological approach. Biol. Philos. 4, 373-405.

Würtz, P., Annila, A., 2008. Roots of diversity relations. J. Biophys. 2008, 8. 